\title{
Analysis of Zhang Hongxing's Clinical Experience in Treating Chronic Cough
}

\author{
Wenguang Zheng, Hongxing Zhang*
}

Dezhou Zhang Hongxing Famous Senior TCM Physician Heritage Studio, Dezhou Hospital of Traditional Chinese Medicine, Dezhou 253000, Shandong Province, China

\begin{abstract}
Director Zhang Hongxing is a famous traditional Chinese medicine (TCM) doctor in Shandong province and a teacher in the Famous TCM Expert Studio in Dezhou city. He has rich clinical experience and considerable experience in the treatment of common clinical chronic coughs. Director Zhang Hongxing believes that chronic cough belongs to the category of "wind cough" and "long-term cough" according to TCM. TCM diagnosis should start from the four aspects of "wind evil residing in lung", "liver", "spleen and stomach", and "yang deficiency". Starting from viewing the human body as an organic whole, distinguish between deficiency or excess in cold and heat, and clinical treatment for cough should focus on dispelling "wind", regulating the functions of liver, spleen, and stomach, and supplementing the body's yang. Formulate treatment based on different categorization, and modify prescription according to the symptoms, and the treatment effects are remarkable.
\end{abstract}

Key words: Chronic cough; Chinese medicine dialectics; Wind cough

Publication date: May, 2021; Publication online: 31 May, 2021

*Corresponding author: Hongxing Zhang, zhxzhx1967@,163.com

\section{Introduction}

Cough is a common symptom of respiratory diseases. A cough that lasts for 8 weeks or more and shows no obvious abnormality in the inflammatory response on chest imaging and blood test is known as chronic cough. Approximately $75 \%$ to $90 \%$ of patients with chronic cough have a definite cause. Modern medicine believes that this is caused by an inflammatory response of the respiratory tract due to the interaction of multiple factors with or without transient airway hyperresponsiveness ${ }^{[1,2]}$. In clinical practice, Western medicine has poor effects on chronic cough which often recurs after stopping using the medicine. Chronic cough seriously affects the quality of life and work efficiency of patients. Therefore, it is imperative to explore active and effective treatment methods. However, TCM often has unique advantages for the treatment of chronic cough. As early as recorded in "Su Wen • Cough Theory", a theory that "(when one coughs) all the internal organs are coughing, not just lungs" was proposed. In his magnum opus "Complete
Works of Jing Yue", Ming dynasty medical scientist Zhang Jingyue divided cough into exogenous and internal injury cough. The diagnosis and treatment based on the holistic approach usually could have ideal effects. The author is fortunate to learn from Director Zhang Hongxing, record clinical cases of his diagnosis and treatment of diseases, and summarize his experience in the diagnosis and treatment of chronic cough from the clinical teaching and medical records as follows.

\section{Disease causes and mechanisms}

\section{1 "Wind evil" residing in lungs}

Coughing is mainly attributed to the lungs. In the early stage of coughing, it is often caused by exogenous infections. At the beginning of the disease, it is necessary to differentiate between exterior and interior site of origin. As stated in the "General Treatise on Causes and Manifestations of All Diseases": "The lungs are in charge of $q i$ together with the body surface, so when 'evil' strikes, the skin will receive it first". And it was said that: "When the lungs suffer from cold, 
it will result in coughing." For coughs caused by the six exogenous diseases, the most common type is "wind evil". The key remote cause of exogenous cough is "wind". The pathological characteristics of "wind evil" are light and constantly changing. When "wind" is in excess, it will be itchy. When the "wind" is the yang "evil", the qi and the body fluid will be easily depleted. Therefore, the "wind evil" can cause dry mouth, itchy throat, nasal congestion, cough and other clinical symptoms. "Wind evil" can often cause diseases combined with cold, heat, and dryness, which should be treated accordingly based on careful diagnosis. Director Zhang believes that wind-induced lung injury persists for a long time, and the "evil" infested lung is still a common cause of chronic cough, so it belongs to the category of "wind cough" and "prolonged cough" of TCM. In clinics, it is very common that patients see a doctor for having cough as the main symptom or the only symptom. According to modern medicine, coughing can be seen in many diseases. Chronic cough persists and does not recover over time, which can be discussed from the perspective of "wind evil" and lungs, but the syndromes and corresponding treatments are ever-changing, and the treatment is still based on syndrome differentiation.

\subsection{Liver and chronic cough}

The liver governs dredging and dissipation, it is a stronghold for regulating $q i$. Stagnation of $q i$ in the liver, reversal and dysfunction of $q i$, and the failure of liver to dredge and dissipate $q i$ can easily make 'earth' element to covert 'metal' element, resulting in cough when it rises to the lungs. From the physiological and pathological characteristics of the two, liver and lungs regulate the rise and descent of the body's qi, with the liver $q i$ rising to the left and the lungs $q i$ descending to the right, thereby maintaining the balance of the $q i$ in human. If the liver $q i$ is in excess and rises too much, it will disrupt the balance of the $q i$ and cause the disorder in rise and descend of the lung $q i$, and the rise of lung $q i$ will induce coughing. Just as You Zaijing writing in the "Jingxianglou Medical Records": "Dry cough and less phlegm are the symptoms of liver $q i$ attacking the lungs, and did not start at the lungs. It is still suitable to treat the liver while nourish lung $q i$ simultaneously".

\subsection{Spleen, stomach and chronic cough}

The spleen and stomach are the regulating hub of the $q i$ in the middle Jiao, the spleen $q i$ is mainly ascending, and the stomach $q i$ declines to harmonize. The spleen and the stomach are adjacent to the lungs, their qi takes different paths but shares the respiratory portal. If the 'evil' invades the spleen and stomach, the ascension and descension of $q i$ in spleen and stomach becomes unbalanced, which can affect the lung's functions of suppressing and sinking qi, causing the lung $q i$ to rise and resulting in cough. According to the Five Elements (Wu Xing) theory, the spleen and lungs belong to the category of 'earth', and the lungs belong to 'metal'. As 'earth' produces 'metal', hence the spleen and lungs are in mother-child relationship, and whether the lungs' $q i$ is sufficient or not depends on whether the spleen's functions of production and conversion are normal. In terms of pathology, as "the spleen is the source of sputum production, and the lungs are the storage site for sputum", clinical manifestations such as cough, sputum expectoration, abdominal distension, and fatigue are often seen. Therefore, clinical treatment usually adopts the philosophy of "fostering earth to produce gold", that is, using drugs that boost the spleen $q i$ to nurture lung $q i$ in order to treat chronic cough at the root causes.

\subsection{Deficiency in qi and yang and chronic cough}

Chronic cough is often accompanied by a deficiency of yang in the organs. "Suwen - Comment on Fever" wrote: "If the 'evil' is gathered together, the $q i$ must be weak." Chronic cough has a long course of disease, the normal $q i$ is deficient and the 'evil' lingers, and the deficiency of lung and spleen $q i$ lead to the 'evils' of 'wind' and cold taking chances to enter the body interior from surface. Deficiency in the normal $q i$ and the firmness of the 'evil' cause the loss of $q i$ and yang. The lingering course of the disease gradually changes from the deficiency of $q i$ in the lungs, spleen and kidney to the syndromes of deficiency in both qi and yang. Currently, in the clinical treatment of chronic cough, the regularly used antibiotics, atomized hormone spray, cold herbal infusion, and the large amount of bitter cold and heat-clearing Chinese medicines used in failed early treatment cause yang qi to become latent as ice and failed to suppress 'evil' $q i$, thereby resulting in lingering chronic cough with complex and ever-changing pathology that is difficult to cure. Therefore, Professor Zhang advocates that the clinical treatment of chronic cough should use drugs that warm the spleen and lungs to supplement the body's yang.

\section{Principles and methods of treatment and}

\section{experience prescriptions}

Director Zhang believed that the lungs were the main organs causing coughing symptoms, and the treatment should be 
focused on the lung system while taking the "wind evils residing in the lungs" into account, the spleen, stomach and the liver system that cause disorder in flowing of $q i$, which was the key factor in chronic cough. The treatment of chronic cough always emphasizes the implementation of the treatment philosophy of regulating $q i$, strengthening the body and eliminating 'evils' throughout the course of treatment. Perform specific diagnosis and be flexible in prescriptions according to the pathological characteristics of chronic cough.

\subsection{Wind evils residing in lungs}

This syndrome is more common in patients who suffer from 'wind evils' for a long time, unhealed for prolonged time, failed to cure and given wrong treatment. For patients with 'wind evils' in the lungs, common symptoms are: daytime cough is the main symptom, frequent coughing after exposure to wind and cold, less coughing at night, nasal congestion, itchy pharynx, hoarse voice, reddish tongue, thin and greasy coating, and slippery pulse. The principle of treatment is to dispel 'wind' and cold, moisten dryness, relieve cough and reduce phlegm: mainly based on modified Maimendong Decoction, and the prescription (glehnia roots, dwarf lilyturf tuber, five-flavor berry, lily, figwort, honey loquat leaves, forsythia, Uncaria, fried burdock seeds, fried perilla seeds, fried perilla stem, siler, smoked plum, Solomon's seal, and fried gardenia) nourishes yin and the lungs, regulates $q i$ and relieves cough.

\subsection{Liver and chronic cough}

Liver dysfunction and liver $q i$ stagnation cause imbalance in liver $q i$, which rise up to attack the lung; Common symptoms are mainly coughing with little or no sputum, that easily affected by emotional changes, bitter mouth, upset, distension of hypochondrium, occasional gastric acid reflux, heartburn, belching, yellow urine, red tongue, and rapid pulsing. Treatment principles: clearing the liver and purging the fire, soothing the liver and relieving cough. The modified Xiaoyao powder and Xiebai powder are prescribed for treatment, prescriptions (Bupleurum, dong quai, poria, white peony, Baizhu (Atractylodes macrocephala), skullcaps, morus bark, lycium bark, Japonica rice, licorice, Uncaria, Forsythia). Xiaoyao powder is used for dredging liver and relieving depression, replenishing liver blood, and aiding the liver; Xiebai powder reduces lung fire, and relieves cough; coordinates the ascension and descension of $q i$ and removes cough. In this prescription, Uncaria and forsythia are empirical medicines. Uncaria can relieve the liver and resolve depression, suppress liver yang and dissipate fire. Forsythia can detoxify and dissipate the heat in middle and upper Jiao, regulates the circulation of $q i$ and relieves cough right at the root cause.

\subsection{Spleen, stomach and chronic cough}

The spleen governs circulation and transformation of $q i$, and when the spleen $q i$ is sufficient, the lung $q i$ is strong, and the deficiency of lung $q i$ is mostly related to the weak spleen. Common symptoms are: cough is obvious in the morning, with more sputum, white phlegm is more common, and often accompanied by fatigue, abdominal distension, diarrhea, sticky mouth, anorexia, sticky stool, fat and large tongue, tooth marks on feces, less tongue coating; and weak pulse; treatment principles: invigorate the spleen, nourish lungs, relieve cough and reduce phlegm; prescribe Erchen Decoction and Liujunzi Decoction; prescriptions (Dang Shen, poria, Baizhu, coix seed, amomum, lotus seed, platycodon, white lentils, Cangzhu (atractylodes lancea), foxnut, tangerine peel, pinellia); Liujunzi Decoction invigorates the spleen and nourishes $q i$, builds up and transports the $q i$, promotes $q i$ and reduces phlegm, Erchen Decoction invigorates the spleen, nourishes $q i$ and relieves cough;

\subsection{Deficiency in yang and chronic cough}

The patient's innate physique is not sufficiently strong, deficient in yang $q i$, and is prone to external attacks by 'wind' and cold. In the later stage, the disease is prolonged and difficult to heal. Common symptoms are: coughing, lethargic, coughing white and sticky sputum, cold limbs, prone to catching cold repeatedly, conditions are aggravated when environment is cold, pale tongue, white tongue coating, weak pulse. Treatment principles: warming the lungs and relieving cough, nourishing the lungs and replenishing the kidneys, keeping the $q i$ of ying and wei in balance; prescribe modified Yupingfeng powder and Erxian Decoction; prescriptions (astragalus, Baizhu, siler, curculio, epimedium, cassia twig, white peony, jujube). If the deficiency of kidney $q i$ is significant, Jinkui Shenqi Pill can be prescribed in addition accordingly.

\section{Examples of cases}

Wang, male, 56 years old, first visited the clinic on May $10^{\text {th }}$, 2018. After catching a cold 3 months ago, he developed symptoms of cough and white sputum that was hard to be coughed out, and body aches, dizziness and discomfort as well as about $38^{\circ} \mathrm{C}$ body temperature. His chest radiograph 
showed probability of bronchitis. White blood cells and neutrophils were increased according to the regular blood test. The patient was told to take cephalosporin antibiotics, atomized spray, and drink plenty of water and other symptomatic treatments to relieve the body soreness, bring the body temperature down, and reduce the sputum significantly. However, cough relief was not obvious, dry cough without sputum, occasional sputum but was low in volume, sticky and filamentous, uncomfortable phlegm, itchy and dry throat, dry lips and nose, dry mouth, foreign body sensation in the throat, aggravated by smoke and cold air irritation, nasal congestion, narcosis, dry stool, poor sleep quality (cannot fall asleep due to itchy throat and cough), red tongue with less fluid, thin yellow tongue coating, and floating pulse. Re-examination of chest X-ray revealed: no obvious abnormalities; re-examination of regular blood showed no obvious abnormalities; the etiology and pathology were 'wind evil' invading the lungs, 'evil' endured, depletion of $q i$ and $y i n$, yin deficiency and fire excess. In addition, the administration of antibiotics and atomized drugs depleted the yang energy, and the normal qi became deficient and the 'evil' lingered. Syndrome type: 'wind evil' invades the lung, yin deficiency and dry cough. Recommended to treat by dispelling the 'wind' and clear the lungs, moisten dryness and relieve cough.

Prescription was modified Shashen Maidong Decoction (Decoction of Glehnia Root and Dwarf Lilyturf Tuber) in combination with the Baihe Gujin Decoction (Lily Bulb Metal-Securing Decoction), recipe: glehnia $30 \mathrm{~g}$, dwarf lilyturf tuber $30 \mathrm{~g}$, five-flavor berry $12 \mathrm{~g}$, lily $30 \mathrm{~g}$, figwort $12 \mathrm{~g}$, honey loquat leaves $30 \mathrm{~g}$, Forsythia $12 \mathrm{~g}$, Uncaria $12 \mathrm{~g}$, fried burdock seed $30 \mathrm{~g}$, fried perilla seed $30 \mathrm{~g}$, fried perilla stem 30 g, siler $15 \mathrm{~g}$, smoked plum $12 \mathrm{~g}$, Solomon's seal 12g, fried gardenia 12g. 7 servings, decocted, 1 serving / day, divided into two portions and taken when warm.

Follow-up visit on May $17^{\text {th }}, 2018$. Patient reported that cough had been relieved, but still suffered from dry mouth and itchy throat. Coughing became frequent when the throat was itchy and coughing stopped when throat was not itchy. Coughing deteriorates when nose was congested and stopped when nose was clear. Previous prescription was modified with Solomon's seal and fried gardenia removed and $10 \mathrm{~g}$ of white silkworm added, and 7 servings were given to the patient.

Third visit to the clinic on May 22 $2^{\text {nd }}, 2018$. Patient reported that dry throat and coughing had improved, but the throat still felt itchy occasionally. After taking 7 servings of the modified prescription issued during the follow-up visit, all symptoms disappeared.

Note: First distinguish between exogenous infection and internal injuries for the cause of cough. Exogenous infections normally have more acute onset, short duration, and are accompanied by exogenous manifestations. Most of them are manifestations of "evil". The main treatment is to evacuate the exogenous pathogens and ventilate the lungs. It is not advisable to use bitter cold, moisturizing, astringent, and antitussive drugs, including the use of too much antibiotics and hormone drugs which can easily cause damage to the body's yang, resulting in deficiency of normal $q i$ and the lingering of 'evil'. When exogenous 'evil' injures the human body for a long time, failure to treat in time and wrong treatment can easily turn it into internal injuries and coughs, further involving organs such as lungs, spleen, stomach, and liver, etc., resulting in long course of disease and difficulty healing. The treatment should mainly focus on regulating visceral qi, blood, yin and yang, and symptomatic treatment. The patient has a long course of disease which belongs to the category of chronic cough. The cough is persistent and difficult to heal, with dry cough and less phlegm as main symptoms, accompanied with deficiency of lung yin, loss of lung moisturization, and abnormal blood pressure. For dry cough, treat by nourishing yin and the lungs to stop coughing, and Professor Zhang will usually prescribe the Shashen Maidong Decoction in combination with the Baihe Gujin Decoction for the patients. In the prescription, glehnia root, dwarf lilyturf, and Solomon's seal serve to nourish yin and the lungs, promote the production of body fluid and relieve cough. Smoked plum and sweet and sour five-flavor berry help resolve yin, astringe the lungs and stop coughing. Lily nourishes the lungs, relieves cough, and calms the mind and spirit. Figwort promotes blood circulation and removes blood stasis, and nourishes blood and yin. Stir-fried gardenia, baba leaves, burdock seeds, stir-fried perilla seeds, and stir-fried perilla stems help to broaden the chest and regulate $q i$, clear the heat from middle and upper Jiao ('burner'), dissipate the 'fire' residing in the lungs, prevent and dispel 'wind', and disperse cold. Uncaria and forsythia are empirical couplet medicines added to stop coughing. If patient has a protracted course, difficulty in stopping cough, and severe cough, add insect medicinal ingredients if necessary to dispel wind, relieve cough and enhance the effects. 


\section{References}

[1] Gong NJ, Lan ZH, Zhu W, Zhang YB, Liu LK. Analysis on TCM master HONG Guangxiang's experience in the treatment of chronic cough [J]. China Journal of Traditional Chinese Medicine and
Pharmacy,2019,34(06):2492-2494.

[2] Lin XJ, Yang Y. Experience of Shashen Maimendong decoction and Ermu powder in treating post-infectious cough [J]. Clinical Journal of Traditional Chinese Medicine,2019,31(12):2247-2250. 